\title{
Interplay between Shape and Roughness in Early-Stage Microcapillary Imbibition
}

\author{
Salvatore Girardo, ${ }^{\dagger}$ Silvia Palpacelli, ${ }^{\S, \|}$ Alessandro De Maio, ${ }^{\S}, \|$ Roberto Cingolani, ${ }^{\perp}$ Sauro Succi, ${ }^{\|}$ \\ and Dario Pisignano*, $,+, \ldots, \#$
${ }^{\dagger}$ National Nanotechnology Laboratory (NNL) of the Consiglio Nazionale della Ricerche (CNR)-Istituto Nanoscienze and
${ }^{\ddagger}$ Dipartimento di Ingegneria dell’Innovazione, Università del Salento, via Arnesano, I-73100 Lecce, Italy
${ }^{\S}$ Numerical Methods Implimentation for Design of Industrial Applications (NuMIDIA) srl, via Berna 31, 00144 Roma, Italy
"Consiglio Nazionale della Ricerche-Istituto di Applicazioni del Calcolo (CNR-IAC), via dei Taurini 9, 00185 Roma, Italy
${ }^{\perp}$ Istituto Italiano di Tecnologia (IIT), via Morego 30, I-16163 Genova, Italy
${ }^{\#}$ Center for Biomolecular Nanotechnologies @UNILE, Istituto Italiano di Tecnologia (IIT), Via Barsanti, I-73010 Arnesano (LE), \\ Italy
}

ABSTRACT: Flows in microcapillaries and associated imbibition phenomena play a major role across a wide spectrum of practical applications, from oil recovery to inkjet printing and from absorption in porous materials and water transport in trees to biofluidic phenomena in biomedical devices. Early investigations of spontaneous imbibition in capillaries led to the observation of a universal scaling behavior, known as the Lucas-Washburn (LW) law. The LW allows abstraction of many real-life effects, such as the

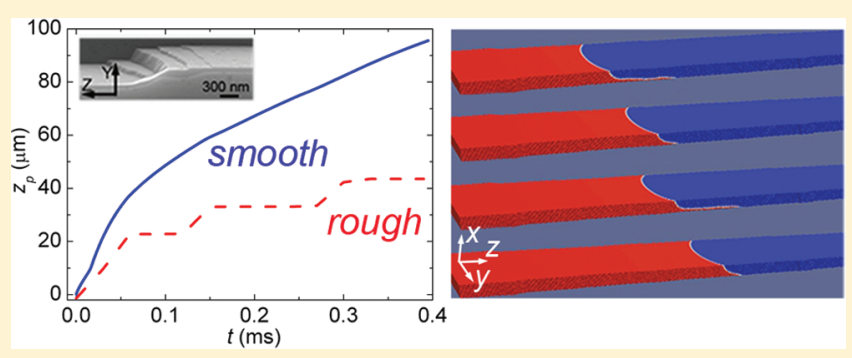
inertia of the fluid, irregularities in the wall geometry, and the finite density of the vacuum phase (gas or vapor) within the channel. Such simplifying assumptions set a constraint on the design of modern microfluidic devices, operating at ever-decreasing space and time scales, where the aforementioned simplifications go under serious question. Here, through a combined use of leading-edge experimental and simulation techniques, we unravel a novel interplay between global shape and nanoscopic roughness. This interplay significantly affects the early-stage energy budget, controlling front propagation in corrugated microchannels. We find that such a budget is governed by a two-scale phenomenon: The global geometry sets the conditions for small-scale structures to develop and propagate ahead of the main front. These smallscale structures probe the fine-scale details of the wall geometry (nanocorrugations), and the additional friction they experience slows the entire front. We speculate that such a two-scale mechanism may provide a fairly general scenario to account for extra dissipative phenomena occurring in capillary flows with nanocorrugated walls.

\section{INTRODUCTION}

Moving fluids at microscopic scales is not an easy task, as it meets with a number of fundamental and practical challenges, with no counterpart in the macroscopic world, among others, the dominance of surface versus volume effects, which implies a steep rise of dissipative phenomena with decreasing size of the devices. Given the paramount role of flow phenomena at the microscale, ways of beating the aforementioned dissipative barrier is a subject of intense research in modern fluid dynamics and applied science in general. Indeed, capillary flows influence a wide class of phenomena in nature and technology. ${ }^{1-3}$ So far, most of these are still described by scientists in terms of the scaling behavior predicted by the Lucas-Washburn (LW) law, ${ }^{4-9}$ which states that the capillary front advances in time along the capillary with a square-root depedence, much slower than ballistic motion. Albeit formally identical to Brownian diffusion, the LW square-root dependence arises from a quite different physical picture, namely, the competition between the capillary drive and the frictional drag against the confining walls. Let $z(t)$ be the coordinate of the moving front at time $t$. Dissipation scales like the front speed $\mathrm{d} z / \mathrm{d} t$ times the linear size, $z(t)$, of the wall region covered by the liquid. The capillary drive, on the other hand, is independent of $z$, since it is localized at the front contact point with the walls. Striking a balance between the two gives $z(\mathrm{~d} z / \mathrm{d} t)=$ constant, yielding the well-known $z \approx t^{1 / 2}$ LW regime. ${ }^{7}$ The LW equation is expected to provide a model valid for sufficiently long times, when only capillary pressure and drag viscous forces are taken into account, whereas both inertial effects ${ }^{8,9}$ and the contact line motion between the gas-solid and liquid phases and its interaction with the roughness of the wetting walls are neglected, ${ }^{10,11}$ i.e., for $t$ much larger than time scales on the order of $\rho R_{1}^{2} / \eta$, where $R_{1}, \rho$, and $\eta$ indicate the hydraulic radius $^{12}$ and the fluid density and dynamic viscosity,

Received: September 8, 2011

Revised: December 15, 2011

Published: January 17, 2012 
respectively. In particular, we recall that $R_{1}$ is given by $2 A / p$, with $A$ the surface area and $p$ the perimeter of the microchannel ( $\mu \mathrm{Ch})$ cross section. For instance, for a $\mu \mathrm{Ch}$ with a rectangular cross-section with base $w$ and height $h, R_{1}=w h /(w+h) .{ }^{12}$

The LW also assumes an idealized global geometry, cylinders or parallelepipeds, ${ }^{13-19}$ supporting a simple Poiseuille flow and straightforward generalizations thereof. However, the early stages of imbibition, when the meniscus takes shape and sets up for motion, are crucially affected not only by inertial effects (unbalance between capillary forces and frictional drag) ) $^{8,9}$ but also by the geometrical and chemicophysical details of the solid walls, such as their microtopographies and degrees of wettability. ${ }^{10,11,20-22}$ These effects lie beyond the simple LW picture, as signaled in the first place by a diverging front speed at $t=0$ and a singular stress tensor at the contact point of the fluid interface with the solid walls. Notwithstanding simulation work $^{23-25}$ and analytical/numerical extensions of the LW analysis, ${ }^{26}$ the general problem of the correlation between friction effects at the nano- and microscale and macroscopic pinning/depinning phenomena induced by wall surface roughness remains open to discussion.

In fact, as the contact line moves in systems of increasingly smaller size, the interaction with the local wall topology plays a crucial role, particularly in the early stage of the penetration, when the flow velocity is at a maximum. ${ }^{18,19}$ A proper account of the solid-liquid interactions at the nanoscale is therefore instrumental to regulate the divergence of the viscous stresses in the vicinity of the contact line, as predicted by classical hydrodynamics. ${ }^{14}$ However, in experiments at imbibition times $t \ll 1 \mathrm{~ms}$, probing the stabilization dynamics of the front remains a challenge. Notwithstanding some pioneering imaging studies, such as the investigation of restriction mapping in fluidic devices with a temporal resolution of $\sim 250 \mathrm{~ms},{ }^{27}$ there is a lack of experimental data, especially at the earliest stage of capillarity in $\mu \mathrm{Ch}$ 's, which are on the other hand of enormous interest for current lab-on-chip (LoC) devices, ${ }^{2}$ where also the imbibition along the corners contributes significantly to the energy dissipation, thus affecting the overall filling dynamics. This may impact relevant LoC applications, including controlling corner-enhanced imbibition phenomena to improve the operation of digital microfluidic chips, ${ }^{28}$ devices for studying the microrheology of viscous fluids based on lithographically made $\mu \mathrm{Ch}$ 's, ${ }^{29,30}$ the early stages of flow dynamics within capillary devices for liquid transport in microsystems, $^{31}$ and autonomous microfluidics relying on spontaneous capillarity, ${ }^{12}$ besides affecting the performances of lithographic technologies such as soft molding ${ }^{32}$ and micromolding in capillaries. ${ }^{33-35}$

Here, we investigate the very early dynamics of capillarity in randomly nanostructured $\mu \mathrm{Ch}$ 's with hydraulic radius $R_{1} \cong 1$ $\mu \mathrm{m}$ in the first hundreds of microseconds. Our high time resolution $(10 \mu \mathrm{s})$ permits, for the first time, analysis of the short-term imbibition in these systems, evidencing the dynamics of the meniscus stabilization and the influence of the dynamic contact angle. By controlling the sidewall roughness, we demonstrate its influence on the evolution of the imbibition dynamics toward the time-asymptotic LW behavior. Indeed, the retarded meniscus stabilization and capillary filling can be attributed to the dynamic contact angle behavior and to the dissipation effects during imbibition, which appear to be dominated by solid-liquid interfacial effects over bulk viscous dissipation in the first tens or hundreds of microseconds. When both experiments and theory meet with limitations, tailored computer simulations may offer an alternative to glean further understanding, with their unique capability to single out separate individual contributions to the phenomenon under scrutiny. Our experimental findings are therefore complemented by lattice Boltzmann (LB) simulations s $^{36-39}$ on channels of rectangular and trapezoidal cross sections, both with and without nanocorrugations. The LB simulations highlight the formation of thin liquid films (and a concomitant, significant slowing of the penetration dynamics by adding random roughness at the capillary sidewalls) in the acute corners of $\mu \mathrm{Ch}$ 's with trapezoidal sections. Since no significant slowing is observed with rectangular cross sections, we conclude that most of the additional drag in corrugated trapezoidal $\mu \mathrm{Ch}$ 's is due to the presence of these wedge-wetting layers and their interaction with nanocorrugations of the wall.

\section{EXPERIMENTAL SECTION}

Master templates constituted by $\mathrm{Si} \mu \mathrm{Ch}$ 's with and without random roughness imposed onto the sidewalls are fabricated by photolithography and wet etching. For producing channels with lateral roughness, we use a homemade semitransparent polymer photomask presenting features with lateral roughness of about $1 \mu \mathrm{m}$. A layer of photoresist $(\mathrm{AZ} 5214 \mathrm{E})$ is then deposited onto a $\mathrm{Si} / \mathrm{SiO}_{2}$ substrate by spin-coating (4000 rpm for $40 \mathrm{~s}$ ). After the thermal curing of the resist at $120^{\circ} \mathrm{C}$ for $1 \mathrm{~min}$, and the alignment between the substrate and the mask, the resist is exposed to UV light for $5 \mathrm{~s}$. Upon photoresist reversal by curing at $112{ }^{\circ} \mathrm{C}(2 \mathrm{~min})$ and UV exposure $(27 \mathrm{~s})$, the pattern is developed $(1 \mathrm{~min})$, and the resist is removed from the exposed regions. Then the $\mathrm{SiO}_{2}$ layer is etched by immersion in $\mathrm{HF}$ solution ( $3 \mathrm{~min}$ ). After subsequent photoresist lift-off, Si etching is carried out by $\mathrm{KOH}$ solution for $10 \mathrm{~s}$ at $110{ }^{\circ} \mathrm{C}$, thus obtaining a $\mu \mathrm{Ch}$ depth of about $1.3 \mu \mathrm{m}$. Finally, the $\mathrm{SiO}_{2}$ layer present on the top of the $\mu \mathrm{Ch}$ is removed by a second HF etching step ( $3 \mathrm{~min}$ ). The same lithographic procedures are employed for creating $\mu$ Ch's with smooth walls by using a photomask with $20 \mu \mathrm{m}$ wide channels without lateral roughness. In this case, the channel height is about $1.5 \mu \mathrm{m}$. The realized $\mathrm{Si} \mu \mathrm{Ch}$ 's exhibit a trapezoidal profile due to the anisotropy of the $\mathrm{KOH}$ etching, whose etch rates depend on the orientation of the crystallographic planes of the Si wafer. Indeed, monocrystalline (100) silicon samples are etched along $\{111\}$ walls inclined at $54^{\circ}$ with respect to the bottom of the $\mu \mathrm{Ch},{ }^{29}$ thus producing master cross sections with isosceles trapezoidal profiles.

The Si masters are used as starting templates for the realization of elastomeric replicas by soft lithography through in situ polymerization $\left(140{ }^{\circ} \mathrm{C}, 15 \mathrm{~min}\right)$ of poly(dimethylsiloxane) (PDMS; Sylgard 184 by Dow Corning, Midland, MI; A:B = 1:9). Samples are realized by the conformal contact of a PDMS replica of the template $\mu \mathrm{Ch}$ 's and a flat elastomer layer deposited on glass by spin coating. In this way, we obtain $\mu$ Ch's with a top base $\left(w_{\mathrm{m}}\right)$ in the range of $10-20 \mu \mathrm{m}$, a bottom base $\left(w_{\mathrm{M}}\right)$ of $12-22 \mu \mathrm{m}$, and a height $(h)$ of $1.3-1.5 \mu \mathrm{m}$. Also, for such final $\mu \mathrm{Ch}$ 's, the sidewalls are at $54^{\circ}$ with respect to the bottom base and at $126^{\circ}$ with respect to the top base (cross-section schematics in Figure 1a). By this fabrication method, we intentionally obtain a random roughness on the lateral walls. We analyze the resulting profile by scanning electron microscopy (Figure 1b), investigating in detail the roughness on the lateral walls (inset of Figure $1 \mathrm{~b}$ ), which exhibit nanofeatures distributed according to the profile shown in Figure 1c.

Filling is performed by releasing a $2 \mu \mathrm{L}$ ethanol (liquid viscosity $\eta=$ $1.2 \times 10^{-3}(\mathrm{~N} \mathrm{~s}) / \mathrm{m}^{2}$, density $\rho=790 \mathrm{~kg} / \mathrm{m}^{3}$, liquid-vapor surface tension $\sigma=22.3 \times 10^{-3} \mathrm{~N} / \mathrm{m}$ ) droplet at the $\mu \mathrm{Ch}$ inlet and analyzed by using an inverted microscope equipped with a Photron Ultima APX-RS fast camera (schematics of the used setup in Figure 2a). A $60 \times$ magnification oil objective is employed, with an acquisition frame rate as high as $10^{5} \mathrm{fps}$ (frames per second), providing for the first time a temporal resolution of $10 \mu \mathrm{s}$ for the investigation of these microfluidic systems in the early stage of capillary penetration. The liquid penetration coordinate, $z(t)$, is measured in the central point of the meniscus formed by liquid wetting the walls at each time $t$ (Figure 


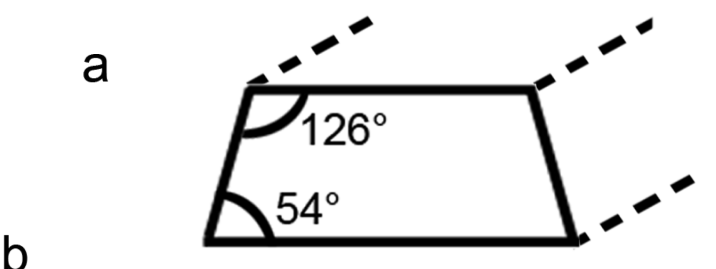

b

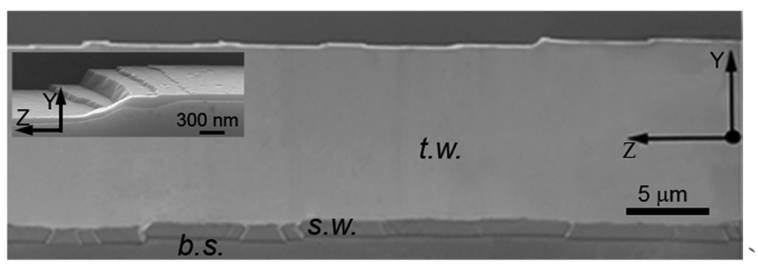

C

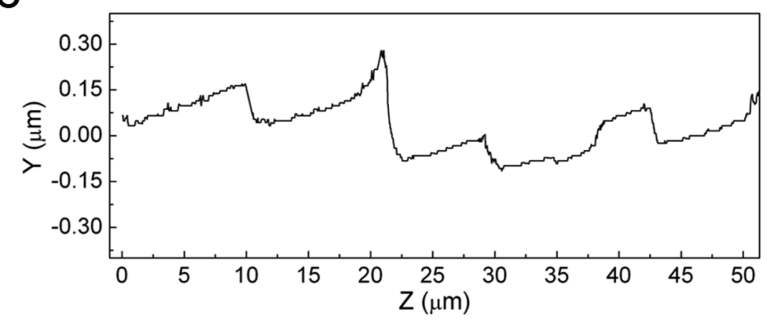

Figure 1. Experimental schematics and existence of two length scale geometries in capillaries. (a) Cross-sectional scheme of the fabricated $\mu$ Ch's. (b) Scanning electron micrograph of the $\mu \mathrm{Ch}$ template with a trapezoidal cross section (top view): t.w. = top wall, s.w. = sidewall, b.s. $=$ bottom surface. On the sidewalls, features in the range of 100-600 and sub-100 $\mathrm{nm}$ are present (inset). (c) Example of the roughness profile of the lateral walls in $\mu \mathrm{Ch}$ 's: $Z=$ channel length coordinate, $Y=$ transversal (width) coordinate.

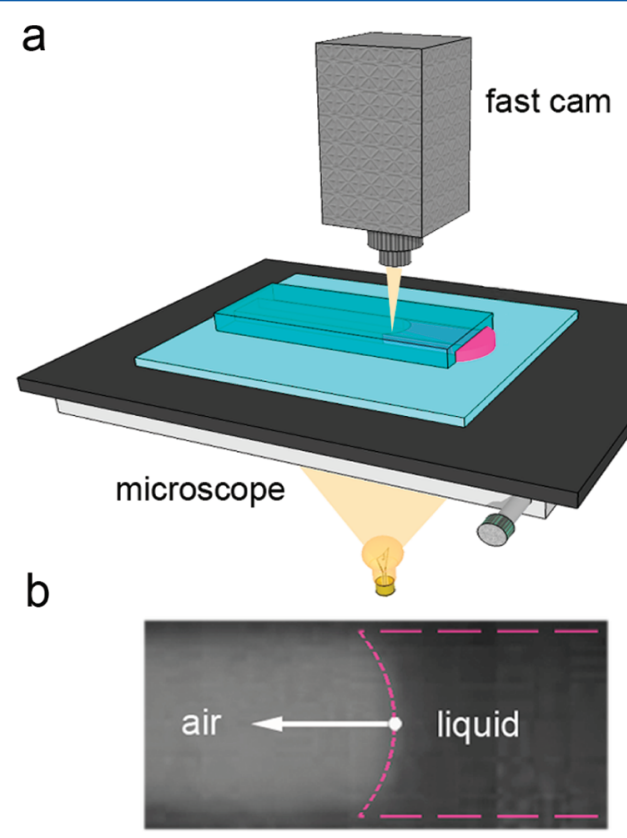

Figure 2. (a) Scheme of the $\mu \mathrm{Ch}$ probing setup. (b) Snapshot micrograph of the liquid motion into a random nanostructured $\mu \mathrm{Ch} . z$ is measured in the central point of the liquid meniscus.

$2 \mathrm{~b}$ ), with an experimental error of $\sim 0.6 \mu \mathrm{m}$, due to the resolution of our optical imaging system. On free and smooth elastomer surfaces, we measure a static contact angle as low as $30^{\circ}$ for ethanol sessile drops by a tensiometer.

\section{LATTICE BOLTZMANN SIMULATIONS}

Computer simulations are performed using the LB method for nonideal, interacting fluids. The LB equation, in single-time relaxation form, reads as follows (unit time step for simplicity): ${ }^{40}$

$$
f_{i}\left(\vec{x}+\vec{c}_{i}, t+1\right)-f_{i}(\vec{x}, t)=-\omega\left(f_{i}-f_{i}^{\mathrm{eq}}\right)+F_{i}
$$

where $f_{i}(\vec{x}, t)$ is the probability of finding a particle at lattice position $\vec{x}$ and time $t$ moving with velocity $\vec{c}_{i}, \omega$ is the relaxation frequency, and $F_{i}$ indicates the effect of external or internal sources. In the above, the subscript $i$ labels the discrete directions of motion in the lattice, for the present case a threedimensional cell with 18 neighbors. The local fluid density, $\rho(\vec{x}, t)$, and velocity, $\vec{u}(\vec{x}, t)$, are obtained by simple linear combinations of the discrete Boltzmann distributions, e.g., $\rho(\vec{x}, t)=\sum_{i} f_{i}(\vec{x}, t)$ and $\vec{u}(\vec{x}, t)=\left[\sum_{i} f_{i}(\vec{x}, t) \vec{c}_{i}\right] / \rho$. These quantities determine the local equilibrium distribution:

$$
f_{i}^{\mathrm{eq}}=\rho w_{i}\left(1+u_{i}+\frac{u_{i}^{2}-u^{2} / c_{\mathrm{s}}^{2}}{2}\right)
$$

where $u_{i}=\left(\vec{u} \cdot \vec{c}_{i}\right) / c_{\mathrm{s}}{ }^{2}$ and $\vec{u}$ is the local flow field (the spacetime dependence is omitted for notational simplicity). In eq 2, $w_{i}$ is a set of weights normalized to unity. For the 19-speed lattice used here (18 moving particles plus 1 rest particle), we have $w_{0}=1 / 3, w_{1}=1 / 18$, and $w_{2}=1 / 36$ for the rest particle, speed 1 particles, and speed $\sqrt{ } 2$ particles, respectively.

The left-hand side of the LB equation represents free molecular motion, whereas the right-hand side describes collisional relaxation to a local equilibrium, $f_{i}^{\mathrm{eq}}(\vec{x}, t)$, on a typical time scale $\tau=1 / \omega$, fixing the fluid kinematic viscosity as $\nu=c_{\mathrm{s}}{ }^{2}(\tau-1 / 2), c_{\mathrm{s}}^{2}=1 / 3$ being the square of the sound speed in lattice units. The effect of internal/external forces is represented by the source term $F_{i}(\vec{x}, t) \approx \vec{F}(\vec{x}, t) \cdot \vec{c}_{i}$. For the case of nonideal interacting fluids, this force term is taken in the form proposed by Shan-Chen, ${ }^{40} \vec{F}(\vec{x}, t)=G \Psi(\vec{x}, t) \sum_{i} w_{i} \vec{c}_{i} \Psi$ $\left(\vec{x}+\vec{c}_{i}, t\right)$, where $\Psi(\vec{x}, t) \equiv \Psi[\rho(\vec{x}, t)]$ is a generalized density and $G$ is the coupling strength of the nonideal attractive interactions. For the generalized density, we adopt the customary Shan-Chen form $\Psi(\rho)=1-\mathrm{e}^{-\rho}$, producing a critical point at $\rho_{\mathrm{c}}=\ln 2$ and $G_{\mathrm{c}}=-4$.

The above LB model can be shown to generate a nonideal excess pressure $P^{*}(\rho) \approx G \Psi^{2}(\rho) / 2$ supporting liquid-vapor phase transitions for sufficiently strong coupling $(G<-4$ in lattice units) and a nonzero surface tension $\sigma \approx-G \int\left(\partial_{x} \Psi\right)^{2} \mathrm{~d} x$, where $x$ runs across the liquid/vapor interface. Furthermore, the fluid interactions at solid walls are modeled by imposing a prescribed value of the wall density $\rho_{\mathrm{W}}=\rho+\Delta \rho$, where the sign and strength of $\Delta \rho$ control the contact angle of the interface. Extensions of this fluid-wall boundary condition to deal with corrugated boundaries ${ }^{39}$ are applied for the case of nanocorrugated geometries.

Since the aim of the simulations is to provide a comparison with experimental results, $\mathrm{LB}$ parameters are matched as far as possible with the experimental setting, reproducing the fabricated geometry and the viscosity and liquid-vapor surface tension of ethanol (reported in the the Experimental Section). The experimental profile of the roughness as extracted by image analysis is directly included within the computational model. The following simulation parameter setting is imposed: coupling strength $G=-5$, liquid density $\rho=790 \mathrm{~kg} / \mathrm{m}^{3}$, liquid viscosity $\eta_{\mathrm{LB}}=0.655 \times 10^{-3}(\mathrm{~N} \mathrm{~s}) / \mathrm{m}^{2}$ and surface 
a

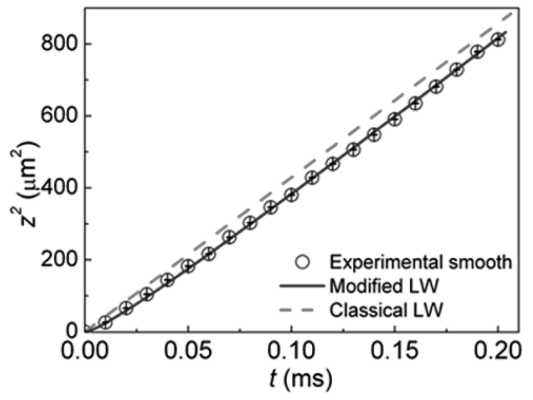

C

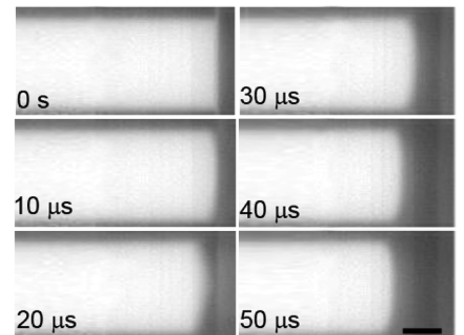

b

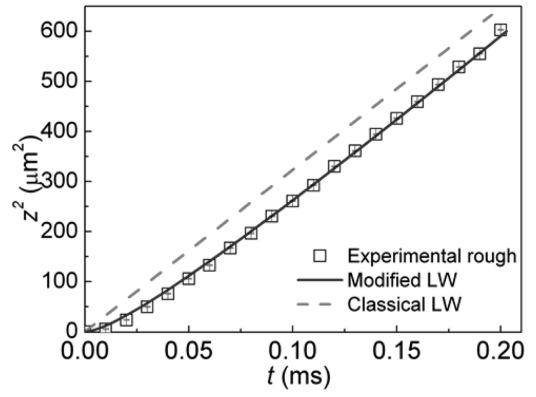

d

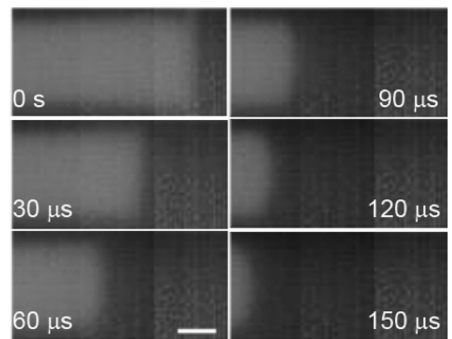

Figure 3. Demonstration of additional friction slowing the entire front with changing meniscus shape during early-stage capillary imbibition. (a, b) Experimental $z^{2}(t)$ data for smooth (circles) and rough (squares) trapezoidal $\mu$ Ch's. Curves are fits by using classical LW (dotted lines) and the molecular kinetics (modified LW) model with friction (eq 7, solid lines). (c, d) Frame of the liquid motion in smooth $(\mathrm{c}, \mathrm{bar}=10 \mu \mathrm{m})$ and in rough $(\mathrm{d}, \mathrm{bar}=5 \mu \mathrm{m}) \mu \mathrm{Ch}$ 's at different time instants.

tension $\sigma_{\mathrm{LB}}=12.2 \times 10^{-3} \mathrm{~N} / \mathrm{m}$. Boundary conditions are enforced at the walls, with a fluid-wall deficit $\Delta \rho / \rho=0.13 \mathrm{~kg} /$ $\mathrm{m}^{3}$. The lattice spacing and time step, which fix the length and time units, respectively, are set to $0.05 \mu \mathrm{m}$ and $0.5 \mathrm{~ns}$. The simulations are run on large grids, with $1200 \times 200 \times 15$ and $1400 \times 114 \times 14$ grid points, for smooth and rough channels, respectively.

\section{RESULTS AND DISCUSSION}

In this section, we first analyze the fast imbibition dynamics and then discuss the interplay mechanism between the $\mu \mathrm{Ch}$ crosssectional shape and nanoscale roughness in determining energy dissipation.

Fast Imbibition Dynamics and the Role of RoughnessInduced Friction. The balance between capillary forces and viscous drag of a liquid front advancing in a rectangular $\mu \mathrm{Ch}$ of width $w$ and height $h$ may be expressed as

$$
\frac{2 \sigma \cos \theta_{0}}{R_{1}}=\frac{8 \eta}{R_{2}^{2}} z \frac{\mathrm{d} z}{\mathrm{~d} t}+\rho\left[z \frac{\mathrm{d}^{2} z}{\mathrm{~d} t^{2}}+\left(\frac{\mathrm{d} z}{\mathrm{~d} t}\right)^{2}\right]
$$

where $\theta_{0}$ is the equilibrium contact angle, $\eta$ is the liquid viscosity, $\rho$ is the liquid density, $\sigma$ is the liquid-vapor surface tension, $R_{1}$ is the hydraulic radius, and the cross-section parameter $R_{2}$ is related to the $\mu \mathrm{Ch}$ geometry $\left(R_{2}=8 h(1-2 \varepsilon)\right.$ $\pi)^{1 / 2} / \pi^{2}$, with $\varepsilon=h / w=0.08-0.13$ for our systems). ${ }^{41,42}$ For capillary filling times $t \gg t_{c, 1}=\rho \tilde{R}^{2} / \eta$ (where we have set $\tilde{R}=$ $\left.R_{2}^{2} / R_{1}\right)$, i.e., for time scales of about $0.5-1 \mu \mathrm{s}$ for our $\mu \mathrm{Ch}$ 's, inertial terms in the previous equation can be neglected, leading to the classical LW relation

$$
z=A \sqrt{t}
$$

with $A=\left[\left(\sigma \tilde{R} \cos \theta_{0}\right) / 2 \eta\right]^{1 / 2}$. We find that, especially for the rough capillaries, such an LW expression is not able to properly fit the experimental $z(t)$ data in the early stages of imbibitions accessible to experimental observation (see Figure 3a,b, where $z^{2}$ data are plotted vs time for better clarity). Note that, given our temporal resolution and $\mu \mathrm{Ch}$ geometry, the condition $t \gg$ $t_{\mathrm{c}, 1}$ is definitely fulfilled; thus, the inertial effects are ruled out. ${ }^{43}$ This is confirmed by visual inspection of the dynamics of the meniscus shape, which exhibits an unexpectedly slow stabilization and evolves from a linear to a curved profile throughout the first $30 \pm 10$ and $120 \pm 30 \mu$ s for smooth and rough capillaries, respectively (Figure $3 \mathrm{c}, \mathrm{d}$ ). Over the same interval, imaging the penetration motion inside the $\mu \mathrm{Ch}$ 's also allows appreciation of the significant variation of the contact angle at the solid-liquid interface.

Moreover, we find a reduction of more than $15 \%$ for the average imbibition velocity in rough $\mu \mathrm{Ch}$ 's with respect to smooth capillaries in the first $400 \mu \mathrm{s}$, which we interpret as mainly due to the change of the dynamic contact angle and the ensuing reduction in capillary pressure. ${ }^{44}$ In fact, an LW expression can still be retained, assuming that the forces on the liquid balance at each time instant $t_{i}^{41,42}$ and introducing an instantaneous, dynamic value of the contact angle $\theta_{t}$. This can be estimated by determining, at each time instant $t_{i}$, a parameter $A_{i}$ fulfilling the expression $z\left(t_{i}\right)=A_{i}\left(t_{i}\right)^{1 / 2}$ by our experimental data and with $A_{i}=\left[\left(\sigma \tilde{R} \cos \theta_{t=t_{i}}\right) / 2 \eta\right]^{1 / 2}$. The resulting $A_{i}$ differ for smooth and rough $\mu \mathrm{Ch}$ 's. Hence, different $\theta_{t=t_{i}}$ values are obtained as $\cos ^{-1}\left(2 A_{i}^{2} \eta / \sigma \tilde{R}\right)$, calculated at each instant. Such a dynamic contact angle $\theta_{t}$ depends therefore on the wetting line velocity (through $A_{i}$ and the $\eta / \sigma$ ratio), thereby developing a space-time dependence during imbibitions and is also found to experimentally vary depending on the wall roughness. For smooth (rough) $\mu \mathrm{Ch}$ 's, $\theta_{t}$ decreases from $74^{\circ}\left(86^{\circ}\right)$ to $62^{\circ}$ $\left(66^{\circ}\right)$ as the liquid penetrates into the $\mu \mathrm{Ch}$ 's and decelerates; i.e., with a reduction, $\Delta \theta_{t}=12^{\circ}\left(20^{\circ}\right)$ over time (Figure 4). It should be noticed that these findings are not contradictory with respect to static values measured by classical tensiometry $\left(\cong 30^{\circ}\right)$, as it is often found that contact angles of liquids within 


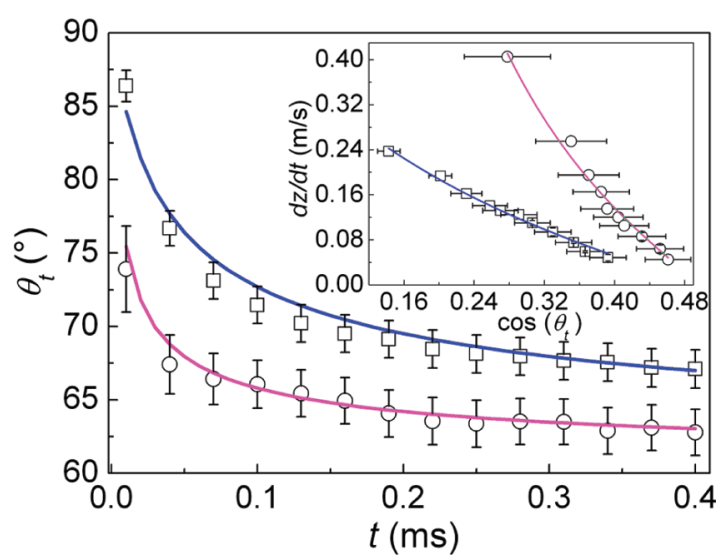

Figure 4. $\theta_{t}$ vs $t$ for smooth (circles) and rough (squares) $\mu$ Ch's and theoretical curves (solid lines) by molecular kinetics (see the text). Inset: wetting line velocity $\mathrm{d} z / \mathrm{d} t$ vs $\cos \theta_{t}$. Solid lines are the best fits to the experimental data by eq 5 , with a displacement frequency parameter $K_{0}$ of about $6 \times 10^{7} \mathrm{~s}^{-1}$ and a $\theta_{0}$ value of $59^{\circ}$. Geometrical parameters for smooth (rough) $\mu$ Ch's are $R_{1}=1.4 \mu \mathrm{m}(1.1 \mu \mathrm{m}), R_{2}=$ $1.2 \mu \mathrm{m}(1.0 \mu \mathrm{m})$, and $\tilde{R}=1.0 \mu \mathrm{m}(0.9 \mu \mathrm{m})$. Liquid viscosity $\eta=1.2 \times$ $10^{-3}(\mathrm{~N} \mathrm{~s}) / \mathrm{m}^{2}$, liquid-vapor surface tension $\sigma=22.3 \times 10^{-3} \mathrm{~N} / \mathrm{m}, k_{\mathrm{B}}$ $=1.38 \times 10^{-23} \mathrm{~J} \mathrm{~K}^{-1}$, and $T=294 \mathrm{~K} . \lambda$ values are in the range of $1-1.5$ $\mathrm{nm}$. Output friction coefficient values are $\zeta_{\text {smooth }}=0.018 \pm 0.003 \mathrm{~Pa} \cdot \mathrm{s}$ and $\zeta_{\text {rough }}=0.042 \pm 0.008 \mathrm{~Pa} \cdot \mathrm{s}$, respectively.

capillaries are significantly different from those of droplets wetting free surfaces. ${ }^{45}$

The dynamic contact angle $\theta_{t}$ can be described by hydrodynamics and molecular kinetics considering dissipation effects during imbibition as due to the viscous friction in the bulk and to the solid characteristics at the moving contact line, respectively. Following Eyring's activated rate theory, ${ }^{46}$ the penetration velocity is given by ${ }^{41,42}$

$$
\frac{\mathrm{d} z}{\mathrm{~d} t}=2 K_{0} \lambda \sinh \left[\frac{\sigma\left(\cos \theta_{0}-\cos \theta_{t}\right)}{2 n k_{\mathrm{B}} T}\right]
$$

where $n$ indicates the number of adsorption sites for the liquid, measured per unit area of the $\mu \mathrm{Ch}$ walls, $\lambda$ is the average distance between adjacent adsorption sites on the capillary walls $\left(n \cong \lambda^{-2}\right), k_{\mathrm{B}}$ is the Boltzmann constant, and $T$ indicates the temperature. In fact, transitory or long-living adsorption phenomena are very likely for the molecules flowing onto complex polymeric surfaces, which exhibit different degrees of nanoscopic corrugation at the length scale typical of polymer molecular dimensions and often nanoporous features, as exploited in many applications including catalysis. ${ }^{47}$ In Figure 4, we plot the theoretical curve resulting from the molecular kinetics model, obtained by following the procedure reported in ref 42. Furthermore, in the inset of Figure 4, we fit the experimental data of the wetting line velocity by eq 5 , i.e., $\mathrm{d} z / \mathrm{d} t$ $=a \sinh \left[b\left(\cos \theta_{0}-\cos \theta_{t}\right)\right]$, in which we set the phenomenological parameters used for fitting, $a=2 K_{0} \lambda$ and $b$ $=\sigma \lambda^{2} / 2 k_{\mathrm{B}} T$. The resulting values for these parameters are $a_{\text {smooth }}=0.18 \pm 0.03 \mathrm{~m} \mathrm{~s}^{-1}, a_{\text {rough }}=0.14 \pm 0.02 \mathrm{~m} \mathrm{~s}^{-1}, b_{\text {smooth }}=$ $7.0 \pm 0.7$, and $b_{\text {rough }}=3.8 \pm 0.3$, respectively. This yields $\lambda$ on the order of the molecular dimension, i.e., $\sim 1 \mathrm{~nm}$, with slightly closer adsorption sites on the capillary walls for rough $\mu \mathrm{Ch}$ 's, which could be related to differences at the nanoscale between surfaces obtained by the different fabrication procedures.

A closer look at friction effects affecting the capillary penetration is achieved by approximating eq 5 as $\mathrm{d} z / \mathrm{d} t=(\sigma)$ $\zeta)\left(\cos \theta_{0}-\cos \theta_{t}\right)$, i.e., introducing the coefficient of wetting line friction (Pa.s) $\zeta=k_{\mathrm{B}} T / K_{0} \lambda^{3}$, where $K_{0}$ is the frequency of liquid molecule displacements at the solid surface. ${ }^{41,42}$ The coefficient $\zeta$ is expected to be from 1 to 3 orders of magnitude larger than the viscosity of the liquid, depending on the relative strength of the solid/liquid and viscous interactions. ${ }^{41,42}$ From our parameters, we find $\zeta_{\text {smooth }}=0.018 \pm 0.003 \mathrm{~Pa} \cdot \mathrm{s}$ and $\zeta_{\text {rough }}$ $=0.042 \pm 0.008 \mathrm{~Pa} \cdot \mathrm{s}$ (inset of Figure 4), indicating that nanoscale roughness acts on the wetting line friction, doubling the value of $\zeta$. The fits, and the observed friction increase in rough $\mu \mathrm{Ch}$ 's with respect to smooth capillaries, indicate that the experimental data are well reproduced by molecular kinetics. ${ }^{41}$ However, we are not able to observe any significant difference between the frequencies of liquid molecule displacements at rough or smooth $\mu \mathrm{Ch}$ 's (the frequency $K_{0}$ being about $6 \times 10^{7}$ $\mathrm{s}^{-1}$ in both the cases). A monotonically decreasing $K_{0}$ upon increasing the root-mean-square surface roughness $r$ is observed on sessile drops on Langmuir-Blodgett films, although with $r$ in a range (about $0.3-1.2 \mathrm{~nm}$ ) of values much lower than in our experiments. ${ }^{48}$

The inclusion of the friction effects, as done by the molecular kinetics model leads to a satisfactory match with experimental data (Figure 3a,b), thus supporting the idea that the slowing of the imbibition dynamics is to be attributed mainly to surface friction effects. In addition, rewriting the previous expression relating the penetration velocity to the dynamic contact angle allows one to highlight a linear dependence of $\cos \theta_{t}$ on the coefficient of wetting line friction, i.e.

$$
\cos \theta_{t}=\cos \theta_{0}-\frac{\zeta}{\sigma} \frac{\mathrm{d} z}{\mathrm{~d} t}
$$

Substituting this expression for $\cos \theta_{t}$ in the force balance with dynamic contact angle $\theta_{t}$, one obtains $\left(2 \sigma / R_{1}\right)\left[\cos \theta_{0}-(\zeta)\right.$ $\sigma)(\mathrm{d} z / \mathrm{d} t)]=\left(8 \eta / R_{2}^{2}\right) z(\mathrm{~d} z / \mathrm{d} t)$, whose integration finally leads to

$$
t=\left(2 \eta / \tilde{R} \sigma \cos \theta_{0}\right) z^{2}+\left(\zeta / \sigma \cos \theta_{0}\right) z
$$

for which friction dominates over bulk viscous dissipation for $z$ $<\zeta \tilde{R} / 2 \eta \cong 8$ and $16 \mu \mathrm{m}$, corresponding to a time crossover $t_{c, 2}$ of 25 and $120 \mu$ s for smooth and rough $\mu \mathrm{Ch}$ 's, respectively, in agreement with the observed experimental dynamics (Figure 3).

Different authors have analyzed the deviations from the LW equation connected to friction forces using various theories of moving wetting lines based on hydrodynamics, ${ }^{49}$ molecular kinetics, ${ }^{50}$ or phenomenology, ${ }^{51}$ in which the dynamic contact angle is described as some function of the wetting line velocity $\mathrm{d} z / \mathrm{d} t$. To date, a universal approach capable of describing all the different situations of the imbibition process is still missing, and consequently, it is not yet fully clear which equation has to be used in connection with the different physical phenomena related to liquid/solid friction properties. Starting from this scenario, modifying the force balance equation has the virtue of being based on a theoretical analysis of energy dissipation, as derived from Eyring's activated rate model. Indeed, this approach exhibits a satisfactory match with our results, which can be related to wetting-wedge layers interaction with the corner geometry as explained in the following.

Dynamics of Wetting-Wedge Layers. The experimentally observed dynamics of the meniscus shape, which evolves toward a profile with a decreasing radius of curvature over the first tens of microseconds, is compatible with the presence of 
wetting fingers along the $\mu \mathrm{Ch}$ corners. Here too thin to be imaged by our experimental setup, such wetting-wedge layers may be visualized using polyurethane prepolymers in PDMS $\mu$ Ch's by optical microscopy ${ }^{52}$ or scanning electron microscopy. ${ }^{33}$ The preferential imbibition along corners within $\mu \mathrm{Ch}$ 's is thoroughly investigated both experimentally and theoretically $^{28,53,54}$ and bears a definite technological relevance, particularly for lithographies based on micromolding in capillaries $^{33}$ and for assessing uncontrolled sample volume escapes in digital microfluidic systems. ${ }^{28}$ From a qualitative viewpoint, corners within $\mu \mathrm{Ch}$ 's represent singularities in the geometrical curvature, altering the force balance between capillarity and dissipation. ${ }^{52,55}$ These imbibition effects along the corners contribute significantly to the overall energy dissipation. Since the effects of roughness on the dissipative mechanisms governing capillary flows is a fundamental open issue in microfluidics and the effects play a key role in applications and processes based on spontaneous imbibition phenomena, investigating liquid layers along wedges and their interaction with rough $\mu \mathrm{Ch}$ walls is a task which warrants indepth investigation. The microscopic interpretation of the deviations from LW scaling in terms of activated rate theory, as given above, accounts for the interpretation of the experimental penetration law $z(t)$, but does not provide any information on morphological microscale features of the advancing front which emerge from nanoscale fluid-wall interactions.

To correlate dissipation effects at the nano- and microscales to possible pinning/depinning of the liquid induced by $\mu \mathrm{Ch}$ roughness, we carried out high-resolution $(0.05 \mu \mathrm{m}$ and $0.5 \mathrm{~ns}$ in space and time, respectively) lattice Boltzmann simulations, ${ }^{56,57}$ covering a time span of about $1 \mathrm{~ms}$. Note that, thanks to the very high time resolution of the experimental data acquisition system, these numerical simulations can be placed in one-to-one correspondence with the experiments, not a common situation in microfluidic simulation. The simulations provide neat evidence of phenomena related to the friction of the moving wetting line. We find good agreement between experiments and simulation, concerning both the filling behavior vs time and the corresponding $\theta_{t}$ values (Figure 5a). In fact, by adding random roughness in the channel sidewalls, the simulations show an average velocity reduction of up to $15 \%$ in the imbibition front. ${ }^{44}$ This reduction is easily evaluated considering the different lengths of smooth and rough microchannels filled by the liquid in $0.4 \mathrm{~ms}$ (Figure 5a). We associate this effect with enhanced energy dissipation due to the formation of liquid layers along wedges and their interaction with rough $\mu \mathrm{Ch}$ walls (Figure $5 \mathrm{~b}$ ).

At the same time, the simulations show the formation of an extended liquid film on the $54^{\circ}$ corners at the bottom of the $\mu \mathrm{Ch}$ (Figure 6a,b). On the basis of the Concus-Finn criterion, $^{58,59}$ the propagation of a liquid on a corner takes place for corner angles below a critical value $\pi-2 \theta_{0}$, in our case roughly $72^{\circ}$. This critical value is consistent with our numerical findings, which show wetting-wedge layers on corners of $54^{\circ}$ and no wetting-wedge layers on corners of $90^{\circ}$ (rectangular cross section) and $126^{\circ} .58,59$ The dynamics and morphology of these layers critically depend on the wall roughness, ${ }^{56,57}$ with a liquid film flowing continuously along the acute corners in the smooth $\mu \mathrm{Ch}$ and growing discontinuously in the rough $\mu \mathrm{Ch}$, with fitful motion (Figure $6 \mathrm{~b}$ ). Indeed, in the latter case, we find that the liquid length at the corners does not grow continuously in time, but alternates between a maximum $(\sim 11 \mu \mathrm{m})$ and a minimum $(\sim 4.5 \mu \mathrm{m})$ value, with distinct time a
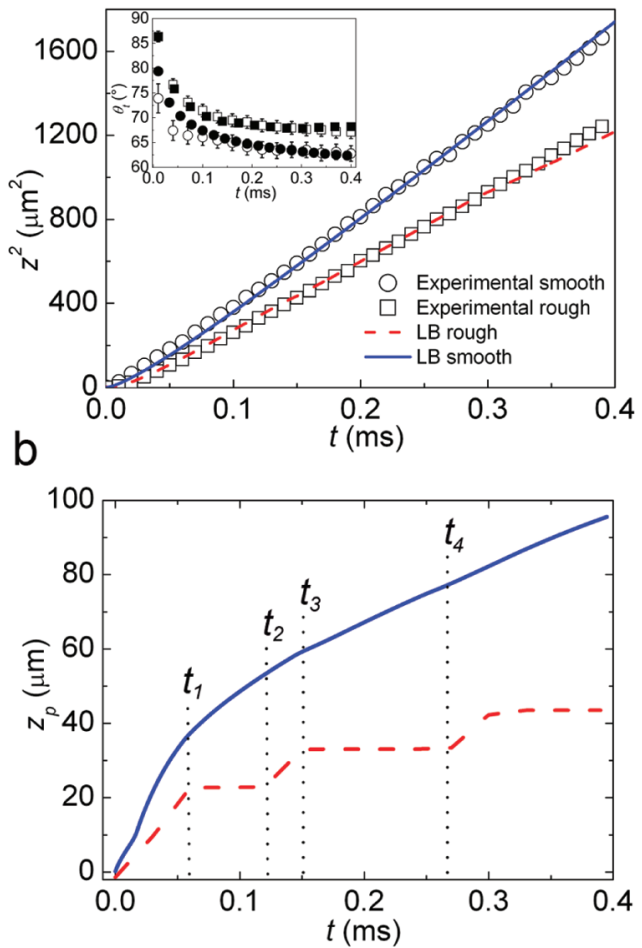

Figure 5. Demonstration of the interaction of wetting-wedge layers with nanocorrugation. (a) Dynamics of the liquid film wetting wedges by $\operatorname{LB} z^{2}(t)$ data (meniscus central point) for smooth (solid lines) and rough (dotted lines) trapezoidal $\mu$ Ch's. Symbols are experimental data on smooth (open circles) and rough (open squares) $\mu \mathrm{Ch}$ 's, highlighting the good agreement between experiments and simulations. Inset: comparison between experimentally determined $\theta_{t}$ (open symbols) and corresponding values obtained by simulations (full symbols) for smooth (circles) and rough (squares) $\mu$ Ch's. (b) Dynamics of the wetting-wedge layer for smooth (solid line) and rough (dotted line) $\mu \mathrm{Ch}$ 's, highlighting the interaction of wettingwedge layers with nanocorrugation. $z_{\mathrm{p}}=$ position of the interface at the corner. $t_{1}=0.06 \mathrm{~ms}, t_{2}=0.12 \mathrm{~ms}, t_{3}=0.15 \mathrm{~ms}$, and $t_{4}=0.27 \mathrm{~ms}$.

gaps during which the liquid film motion is temporarily suppressed (Figure 6b). This stick-slip motion of the front, induced by temporary pinning of the liquid film wetting wedges, leads to a reduction in the penetration length, which is related to the energy dissipation mechanisms described previously in terms of friction effects at the solid/liquid interface. The idea of a relation between the macroscopic, wetting-wedge dynamics and the slowing of the liquid penetration into rough $\mu \mathrm{Ch}$ 's is further corroborated by simulations performed on the rectangular geometry, which show no evidence of layers along corners and only a weak $(<2 \%)$ slowing of the filling velocity for rough $\mu \mathrm{Ch}$ 's with respect to smooth $\mu \mathrm{Ch}$ 's (Figure 7 ). By unveiling a possible correlation between the occurrence and dynamics of the wetting-wedge layers and the degree of friction experienced by the penetrating fluid wall, the simulations provide a significant help in the interpretation of the experimental and theoretical observations.

\section{CONCLUSIONS}

Taken together, our findings suggest that the wetting front advances on a solid surface, with friction properties of the liquid/solid interface dependent on the wall roughness. Such 
a

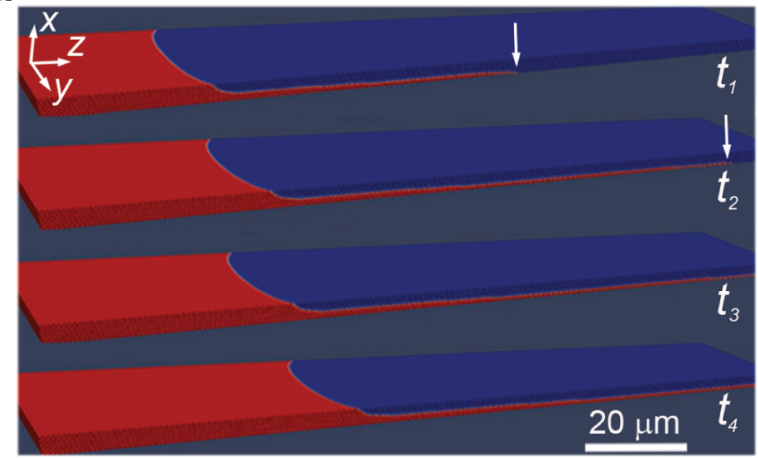

b

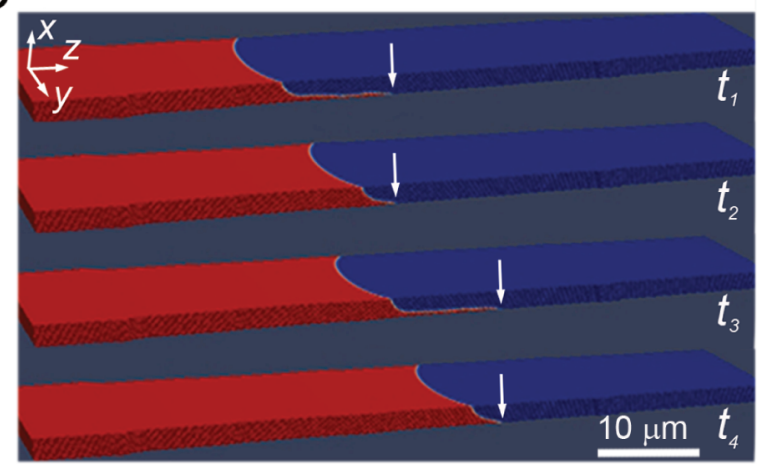

Figure 6. The global geometry sets the conditions for small-scale structures and hence for interplay between shape and roughness. Micrographs of LB simulations: $t_{1}=0.06 \mathrm{~ms}, t_{2}=0.12 \mathrm{~ms}, t_{3}=0.15$ $\mathrm{ms}$, and $t_{4}=0.27 \mathrm{~ms}$ for smooth (a) and rough (b) $\mu \mathrm{Ch}$ 's. The arrows indicate the wetting-wedge layer position along the corners. Different scales are used for the two microchannels to better visualize the meniscus shape in the two cases.

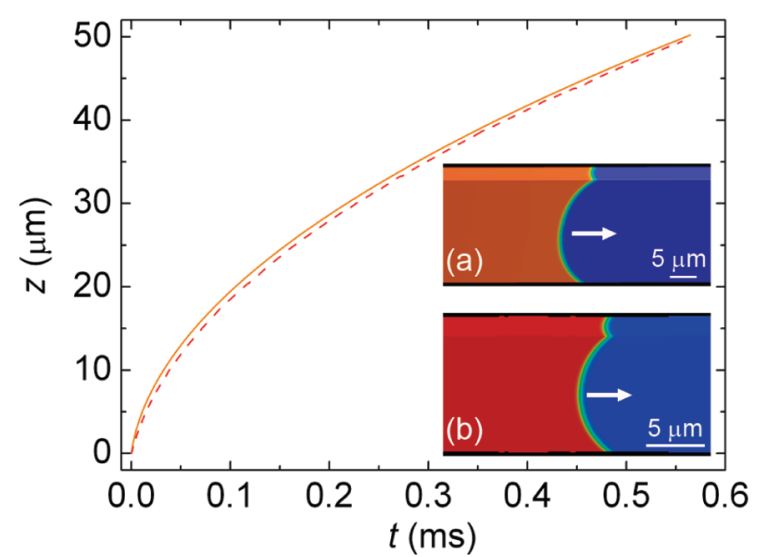

Figure 7. LB $z(t)$ curves for smooth (solid) and rough (dotted) rectangular $\mu \mathrm{Ch}$ 's. Inset: micrographs of LB simulations for smooth (a) and rough (b) rectangular $\mu \mathrm{Ch}$ 's, respectively. The arrows indicate the flow direction.

nanoscopic effects can be described in macroscopic terms as a reduction in capillary pressure depending on the value of the dynamic contact angle, related to the surface friction coefficient $\zeta$ and to the liquid front velocity. At a nanoscopic level, the reduction in penetration height for rough $\mu \mathrm{Ch}$ 's is directly connected to the evolution of a liquid film along the acute corner and its complex interaction with the roughness of the solid walls at the solid/liquid/vapor interface. More precisely, since wetting-wedge layers are fine-scale structures comparable with the heterogeneity scale of the corrugation, they are more sensitive to additional drag, resulting in stick-slip motion and eventually even pinning of the liquid front. The above picture offers an elegant, and possibly paradigmatic, example of a twoscale feedback loop between the micro- and nanoscale physics of early imbibition.

Global geometrical features, i.e., sharp corners, below the critical Concus-Finn angle, ${ }^{58,59}$ promote the formation of finescale structures (wetting layers). With smooth walls these layers do not experience any appreciable extra dissipation as compared to the bulk front; hence, they remain "energetically invisible". In the presence of nanocorrugations, however, they couple strongly to the microgeometrical imperfections and experience a significant extra dissipation, which ultimately results in a slowing of the overall front motion.

It would be interesting to perform LB simulations down to the nanoscopic scale and investigate the possible onset of prewetting layers, as observed recently in molecular dynamics simulations. ${ }^{60}$ As shown in ref 61 , a detailed LB-molecular dynamics comparison requires subnanoscopic spatial resolution, and consequently such prospective simulations will necessarily be restricted to much shorter channels than those considered in this paper.

\section{AUTHOR INFORMATION}

\section{Corresponding Author}

*Phone: +39 0832298104. Fax: +39 0832298146. E-mail: Dario.pisignano@unisalento.it.

\section{ACKNOWLEDGMENTS}

We acknowledge support from the NMP-031980EU EU Project_INFLUS.

\section{REFERENCES}

(1) Wheeler, T. D.; Stroock, A. D. Nature 2008, 455, 208-212.

(2) Squires, T. M.; Quake, S. R. Rev. Mod. Phys. 2005, 77, 977-1026.

(3) Rost, M.; Laurson, L.; Dube, M.; Alava, M. Phys. Rev. Lett. 2007, 98, 0545021-4.

(4) Bell, J. M.; Cameron, F. K. J. Phys. Chem. 1906, 10, 658-674.

(5) LeGrand, E. J.; Rense, W. A. J. Appl. Phys. 1945, 16, 843-846.

(6) Jeje, A. A. J. Colloid Interface Sci. 1979, 69, 420-429.

(7) Washburn, E. W. Phys. Rev. 1921, 17, 273-283.

(8) Quéré, D. Europhys. Lett. 1997, 39, 533-538.

(9) Fries, N.; Dreyer, M. J. Colloid Interface Sci. 2008, 327, 125-128.

(10) Stange, M.; Dreyer, M. E.; Rath, H. J. Phys. Fluids 2003, 15, 2587-2601.

(11) De Gennes, P. G. Rev. Mod. Phys. 1985, 57, 827-863.

(12) Juncker, D.; Schmid, H.; Drechsler, U.; Wolf, H.; Wolf, M.; Michel, B.; de Rooij, N.; Delamarche, E. Anal. Chem. 2002, 74, 61396144.

(13) Courbin, L.; Denieul, E.; Dressaire, E.; Roper, M.; Ajdari, A.; Stone, H. A. Nat. Mater. 2007, 6, 661-664.

(14) Rio, E.; Daerr, A.; Andreotti, B.; Limat, L. Phys. Rev. Lett. 2005, 94, 0245031-4.

(15) Geromichalos, D.; Mugele, F.; Herminghaus, S. Phys. Rev. Lett. 2002, 89, 1045031-4.

(16) Soriano, J.; Mercier, A.; Planet, R.; Hernandez-Machado, A.; Rodriguez, M. A.; Ortın, J. Phys. Rev. Lett. 2005, 95, 1045011-4.

(17) Gruener, S.; Huber, P. Phys. Rev. Lett. 2009, 103, 1745011-4.

(18) Hamraoui, A.; Thuresson, K.; Nylander, T.; Yaminsky, V. J. Colloid Interface Sci. 2000, 226, 199-204.

(19) Stukan, M. K.; Ligneul, P.; Crawshaw, J. P.; Boek, E. S. Langmuir 2010, 26, 13342-13352.

(20) Acquaroli, L. N.; Urteaga, R.; Berli, C. L. A.; Koropecki, R. R. Langmuir 2011, 27, 2067-2072. 
(21) Chibbaro, S.; Costa, E.; Dimitrov, D. I.; Diotallevi, F.; Milchev, A.; Palmieri, D.; Pontrelli, G.; Succi, S. Langmuir 2009, 25, 1265312660 .

(22) Patro, D.; Bhattacharyya, S.; Jayaram, V. J. Am. Ceram. Soc. 2007, 90, 3040-3046.

(23) Kusumaatmaya, H.; Pooley, C. M.; Girardo, S.; Pisignano, D.; Yeomans, J. M. Phys. Rev. E 2008, 77, 0673011-4.

(24) Kusumaatmaja, H.; Yeomans, J. M. Langmuir 2007, 23, 60196032.

(25) Hyvaluoma, J.; Harting, J. Phys. Rev. Lett. 2008, 100, 246001-4.

(26) Chibbaro, S.; Biferale, L.; Diotallevi, F.; Succi, S. Eur. Phys. J. 2009, 171, 223-228.

(27) Riehn, R.; Lu, M.; Wang, Y.-M.; Fang Lim, S.; Cox, E. C.; Austin, R. H. Proc. Natl. Acad. Sci. U.S.A. 2005, 102, 10012-10016.

(28) Andersson, P.; Jesson, G.; Kylberg, G.; Ekstrand, G.; Thorsén, G. Anal. Chem. 2007, 79, 4022-4030.

(29) Girardo, S.; Cingolani, R.; Pisignano, D. Anal. Chem. 2007, 79, $5856-5861$

(30) Girardo, S.; Cingolani, R.; Pisignano, D. J. Chem. Phys. 2007, 127, 164171-5.

(31) Bouaidat, S.; Hansen, O.; Bruus, H.; Berendsen, C.; Kristian Bau-Madsen, N.; Thomsen, P.; Wolff, A.; Jonsmann, J. Lab Chip 2005, $5,827-836$

(32) Persano, L.; Molle, S.; Girardo, S.; Neves, A. A. R.; Camposeo, A.; Stabile, R.; Cingolani, R.; Pisignano, D. Adv. Funct. Mater. 2008, 18, 2692-2698.

(33) Kim, E.; Whitesides, G. M. J. Phys. Chem. B 1997, 101, 855863.

(34) Kim, E.; Xia, Y.; Whitesides, G. M. Nature 1995, 376, 581-584.

(35) Kim, E.; Xia, Y.; Whitesides, G. M. J. Am. Chem. Soc. 1996, 118, 5722-5731.

(36) Benzi, R.; Succi, S.; Vergassola, M. Phys. Rep. 1992, 222, 145197.

(37) Shan, X.; Chen, H. Phys. Rev. E 1994, 49, 2941-2948.

(38) Martys, N. S.; Chen, H. Phys. Rev. E 1996, 53, 743-750.

(39) De Maio, A.; Palpacelli, S.; Succi, S. Commun. Comput. Phys. 2011, 9, 1284-1292.

(40) Succi, S. The Lattice Boltzmann Equation; Oxford University Press: New York, 2001.

(41) Martic, G.; Gentner, F.; Seveno, D.; Coulon, D.; De Coninck, J.; Blake, T. D. Langmuir 2002, 18, 7971-7976.

(42) Martic, G.; Gentner, F.; Seveno, D.; De Coninck, J.; Blake, T. D. J. Colloid Interface Sci. 2004, 270, 171-179.

(43) We also point out that the poor description provided by the classical LW to experimental data cannot be due to the uncertainty in the beginning instant $(t=0)$ of capillary penetration. Indeed, also fitting experimental data by a function of the form $z=A\left(t-t_{0}\right)^{2}$, and including the possibility of an initial temporal offset $\left(t_{0}\right)$, does not provide matching with experiments.

(44) Considering the slight variations among smooth and rough $\mu$ Ch's in terms of hydraulic radius, the observed difference in the imbibition dynamics cannot be ascribed to the different values of the cross-sectional areas.

(45) Ichikawa, N.; Hosokawa, K; Maeda, R. J. Colloid Interface Sci. 2004, 280, 155-164.

(46) Glasstone, S.; Laidler, K. J.; Eyring, H. J. The Theory of Rate Processes; McGraw-Hill: New York, 1941.

(47) Samokhvalov, A.; Tatarchuk, B. J. Catal. Rev.-Sci. Eng. 2010, 52, 381-410.

(48) Semal, S.; Blake, T. D.; Geskin, V.; de Ruijter, M. J.; Castelein,

G.; De Coninck, J. Langmuir 1999, 15, 8765-8770.

(49) Cox, R. G. J. Fluid Mech. 1986, 168, 169-194.

(50) Blake, T. D.; Haynes, J. M. J. Colloid Interface Sci. 1969, 30, 421-423.

(51) Joos, P.; Van Remoortere, P.; Bracke, M. J. Colloid Interface Sci. 1990, 136, 189-197.

(52) Girardo, S.; Cingolani, R.; Chibbaro, S.; Diotallevi, F.; Succi, S.; Pisignano, D. Appl. Phys. Lett. 2009, 94, 171901-3.
(53) Dong, M.; Chatzis, I. J. Colloid Interface Sci. 1995, 172, 278288.

(54) Weislogel, M. M.; Lichter, S. J. Fluid Mech. 1998, 373, 349-378.

(55) Langbein, D. Capillary Surfaces; Springer: Berlin, 2002.

(56) Chibbaro, S.; Biferale, L.; Diotallevi, F.; Succi, S.; Binder, K.; Dimitrov, D.; Milchev, A.; Girardo, S.; Pisignano, D. Europhys. Lett. 2008, 84, 44003-5.

(57) Diotallevi, F.; Biferale, L.; Chibbaro, S.; Puglisi, A.; Succi, S. Phys. Rev. E 2008, 78, 036305-7.

(58) Concus, P.; Finn, R. Proc. Natl. Acad. Sci. U.S.A. 1969, 63, 292299.

(59) Bico, J.; Quéré, D. J. Colloid Interface Sci. 2002, 247, 162-166.

(60) Henrich, B.; Cupelli, C.; Santer, M.; Moseler, M. New J. Phys. 2008, 10, 113022 .

(61) Horbach, J; Succi, S. Phys. Rev. Lett. 2006, 96, 224503. 